\title{
Characterization of Fractal Concentrators of Light at High Concentrations
}

\author{
Ilia Katardjiev \\ Department of Electrical Engineering, Uppsala University \\ ilia.katardjiev@angstrom.uu.se \\ 15 February 2021
}

\section{Abstract}

A numerical characterisation of a fractal concentrator of light at 100X is presented. It is shown that such concentrators exhibit sufficiently large acceptance angles, excellent exit flux uniformity within the acceptance range and transmittance generally exceeding $85 \%$.

\section{Introduction}

Fractal concentrators (FC) have been recently proposed[1] for light concentration. They represent an array of elongated optical elements (OE) of general conical shape separated by small gaps of a lower refractive index optical material (typically air) and enclosed at the periphery by a reflecting mirror structure as illustrated in $2 \mathrm{D}$ in Figure $1 \mathrm{a}$. Consequently, rays entering an OE initially propagate through total internal reflection until they eventually lose radial component and start bifurcating through concurrent refraction/reflection events at the interfaces creating in the process a cascade of fractal rays. The radial component is defined as the projection of the ray's unit vector onto the radius vector connecting the momentary ray's position with the virtual origin of the concentrator defined by the common intersection point of the mirror planes. Thus, most generally, such concentrators operate under the principle "one ray in - more than one fractal ray out". Focusing is primarily achieved through the partial overlap of the so-called "diffuse images"[1] of the individual OEs which figuratively speaking represent the projection of the envelope of the fractal cascades created by all rays entering an OE within a given acceptance angle onto the focal plane. It has also been shown[1] that the size of the "diffuse images" is a monotonically increasing function of the OE's apex angle. Hence, small apex angles result in small "diffuse images", i.e. better focusing and lower rejection.

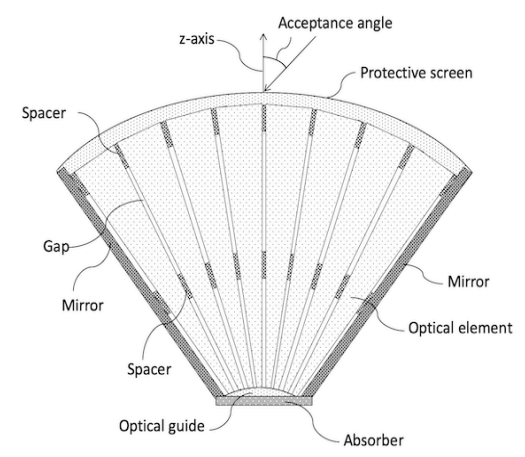

Figure 1a. Schematic of a fractal concentrator

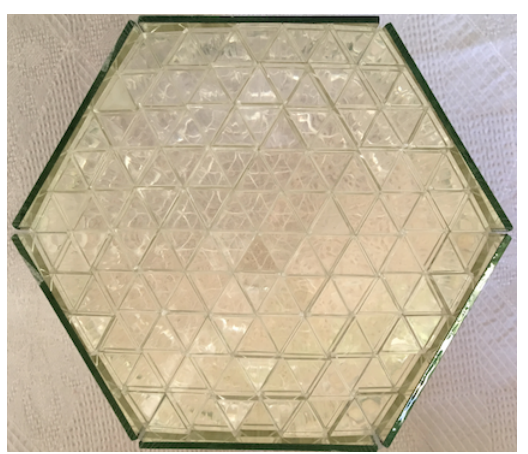

Figure 1b. Top view of a fractal concentrator 
Fig. 1b shows a top view of a FC concentrator. Preliminary experimental work[2] with concentrators at $\mathrm{Cg}=3 \mathrm{X}$ indicates that, indeed, fractal concentrators operate as theoretically predicted[1]. The OE's geometry can vary depending on the specific design. At low geometrical concentrations FC concentrators are shown to have a sufficiently large acceptance angle eliminating thus the need for sun tracking. This is not the case at high concentrations. Thus, the acceptance angle $\theta_{a}$ of the concentrator most generally is given by:

$\theta_{a}=\operatorname{asin}\left[\frac{n_{1}}{n_{2} \sqrt{C_{g}}}\right]-\varepsilon=\theta_{\text {th }}-\varepsilon$

where $n_{1}$ is the refractive index of the OEs, $n_{2}$ is the refractive index of the gaps, $\varepsilon$ is a function of the angular aperture of the concentrator and those of the OEs as well as geometry, while $\theta_{\text {th }}$ represents the theoretical limit. Strictly speaking, the angular acceptance aperture of the concentrator represents the common intersection between the angular acceptance apertures of the individual OEs. Since, as discussed above, small apex angles of the OEs result in small rejection it is expected that small apex angles of the concentrator itself would result in larger acceptance angles. In practice, $\theta_{\mathrm{a}}$ is readily calculated numerically for any specific geometry.

It is the objective of this work to study numerically fractal concentrators at a geometrical concentration $\mathrm{C}_{\mathrm{g}}$ of $100 \mathrm{X}$. Emphasis lies on the acceptance angle as well as on the uniformity of the concentrated flux.

\section{Concentrator design}

The concentrator consists of six OEs the latter representing truncated triangular pyramids with a conical cross-section such that the ratio between the front (top) and the back surfaces defines the targeted geometrical concentration ratio $C_{g}$. Thus, the front surfaces of the OEs are slightly tilted from each other and form a hexagonal input aperture. The OEs are further assumed to be made of an optical material with a refractive index of 1.583. Next, the front surfaces represent equilateral triangles with a side of $12 \mathrm{~mm}$ resulting in a length of the backside of $1.2 \mathrm{~mm}$ at a concentration of 100X. The height of an OE is chosen to be $112 \mathrm{~mm}$. These dimensions result in a sufficiently small apex angle of the OEs to guarantee relatively small "diffuse images" as discussed above. Thus, the resulting half apex angle $\alpha / 2$ of the concentrator is $4.77^{\circ}$.

Further, the chosen geometry of the OEs also guarantees that the angle $\zeta$ between two neighbouring walls of an OE satisfies the following inequalities:

$\pi / 2-\theta_{c}<\zeta<2 \theta_{c}$

where $\theta_{c}$ is the critical angle of total internal reflection (TIR) given by $\theta_{c}=\operatorname{asin}\left(n_{1} / n_{2}\right)$.

The importance of inequalities (2) is that they guarantee[1] the existence of a non-zero angle $\theta_{\text {cut-off }}$ given by 
where

$$
\omega\left(\theta_{c}, \zeta\right)=\operatorname{asin}\left[\sin \left(\theta_{c}\right) \sqrt{1-\cot ^{2}\left(\theta_{c}\right) \cot ^{2}(\zeta)}\right], \quad \text { for } \pi / 2-\theta_{c} \leq \zeta \leq \zeta_{\text {opt }}
$$

and

$\omega\left(\theta_{c}, \zeta\right)=\operatorname{acos}\left[\cot \left(\theta_{c}\right) \frac{1-\cos (\zeta)}{\sin (\zeta)}\right], \quad$ for $\zeta_{o p t} \leq \zeta \leq 2 \theta_{c}$

and where

$\zeta_{\text {opt }}=\operatorname{acos}\left[1-\sin \left(\theta_{c}\right)\right]$

such that when the ray's radial component $\cos (\pi / 2-\beta)$ at a given impact point with a sidewall becomes smaller than $\cos \left(\pi / 2-\theta_{\text {cut-off }}\right)$ then the ray is "forcibly" [1] refracted out of the OE in the subsequent collision event ${ }^{1}$. This results in an accelerated avalanching of the fractal cascade[1] and hence in the additional homogenisation of the exit flux.

Next, the gaps between the sidewalls of the OEs at the top surface are assumed to be 0.1 $\mathrm{mm}$ and decrease proportionally with height to conserve $C_{\mathrm{g}}$. In other words, the gap widths need not be uniform and are kept as small as possible but still large enough to guarantee the validity of geometrical optics. The refractive index of the gaps is assumed to be 1 . The absorption coefficient within the $\mathrm{OE}$ is initially assumed to be zero for clarity of the discussion but a typical example is given below for illustration.

A note on the gap width. In the above design two sets of spacers were used to define the gaps, namely one at the front surface of the OEs and one at some distance from the first set. The positioning of the second set may also be at the focal plane. The use of two sets of spacers generally is superfluous since only one set is sufficient to define a gap between two planar surfaces. Since light entering the concentrator through the gaps is generally lost it is advantageous that the first set of spacers is omitted and thus reduce the unnecessary loss of intensity. The slope of the sidewalls of the OEs at the entrance in such cases may need to be modified in order to guarantee a sufficient gap width. In other cases, it may be advantageous to omit the second set of spacers.

\section{Numerical evaluation}

The optical performance of the concentrator described above is simulated through standard ray tracing employing Fresnel refraction and reflection at interfaces. For each angle of incidence 600000 rays randomly distributed over the acceptance surface (excluding the gaps) have been traced with a precision better $0.1 \%$ meaning that at least $99.9 \%$ of the energy in each fractal cascade is traced to the end. The angle of incidence $\theta$ is measured with respect to the central axis of the concentrator. The peripheral mirrors are assumed to

\footnotetext{
${ }^{1}$ Strictly speaking this statement is mathematically true when the radius vector is approximately parallel to the edge between two neighbouring sidewalls but due to the large height to width aspect ratio of the $O E$ it is practically true for any impact point with the sidewall.
} 
be made of Aluminium. The reflection coefficient for Al was taken from Hébert[3] assuming a refractive index of 1.3 and an extinction coefficient of 7.46 for a wavelength of $619 \mathrm{~nm}$.

The transmittance as a function of the incidence angle is shown in Figure $2 a$.

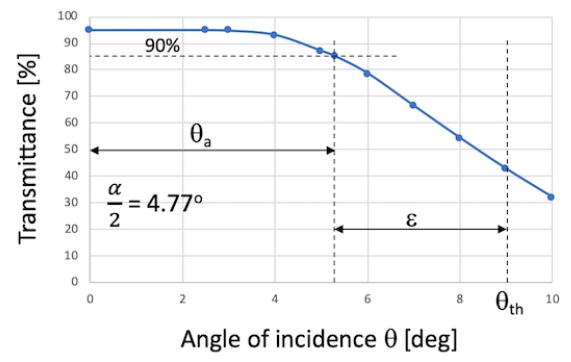

Figure 2a. Transmittance for $\alpha / 2=4.77^{\circ}$

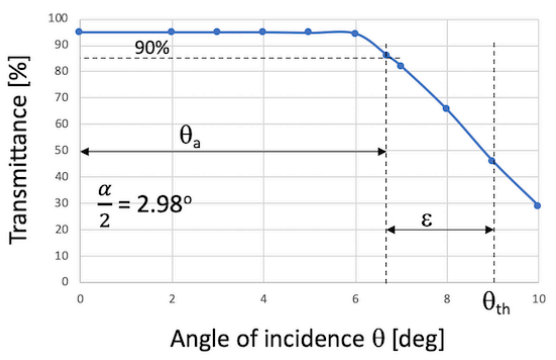

Figure $2 b$. Transmittance for $\alpha / 2=2.98^{\circ}$

From the latter it follows that $\theta_{\mathrm{a}} \approx 5.3^{\circ}$ and $\varepsilon \approx 3.8^{\circ}$. The loss of $5.1 \%$ within the acceptance range is mostly due to reflection off the top surface as the mirror losses become increasing around the region where the transmission starts to decline. From the above discussion it is expected that decreasing the half apex angle $\alpha / 2$ of the concentrator would result in an improved transmission. Indeed, Figure $2 b$ confirms this expectation. The height of the OEs in this case is increased from $112 \mathrm{~mm}$ to $180 \mathrm{~mm}$ resulting in $\alpha / 2=2.98^{\circ}$ while keeping all other dimensions unchanged. From Fig. $2 \mathrm{~b}$ one deduces $\theta_{\mathrm{a}} \approx 6.7^{\circ}$ and $\varepsilon \approx 2.4^{\circ}$. The conclusion from this exercise is that one can tune the acceptance angle to a desired value within obvious constraints.

In practical situations fractal concentrators are to be used in a flat panel configuration as illustrated in Figures 3a,b.

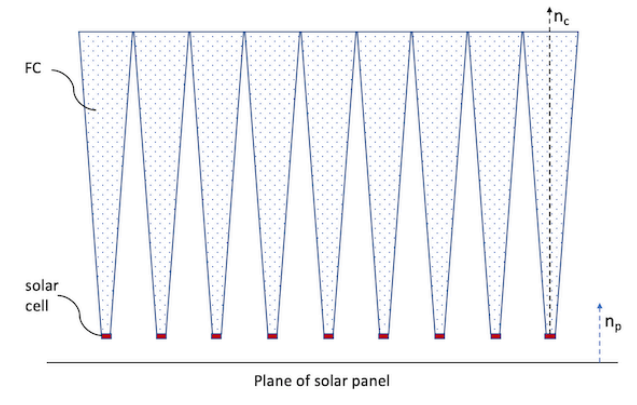

Figure 3a. Sideview of a FC solar panel

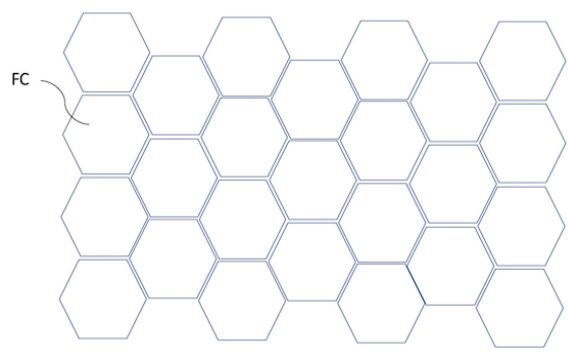

Figure 3b. Top view of a FC solar panel

Specifically, the individual FC concentrators are tightly arranged in a planar array such that their axes are parallel to each other and to the normal to the panel. Referring back to Figs. $2 a, b$ it is noted that the transmission outside the acceptance angle exhibits a steady decline. This behavior may be used to one's advantage to implement sun tracking[4] by tweaking the tilt of a small number of FCs in the panel without the need for additional tracking sensors and without sacrificing performance.

We now take a look at the uniformity of the exit flux for the case in Fig. $2 b$. The calculations indicate that the probability density distribution of the exit flux at the focal plane is practically flat up to incidence angles of $5^{\circ}$ where a detectable non-uniformity is observed as illustrated in Figures $4 a, b$. The latter display the probability distribution at two different viewing angles. The red arrow in Fig. 4a indicates the azimuthal direction of the incident rays. The intensity within the gaps has been discarded to outline the position of the OEs. It is pointed out that the transmittance at this point is unaltered indicating that the non-uniformity is caused by an internal redistribution of the exit flux. The explanation is that 
due to the larger angle of incidence, the volume of the fractal cascades in the OEs farthest along the azimuthal direction is larger than the corresponding volume in neighboring OEs resulting in a net outflux of intensity from the farthest OEs.

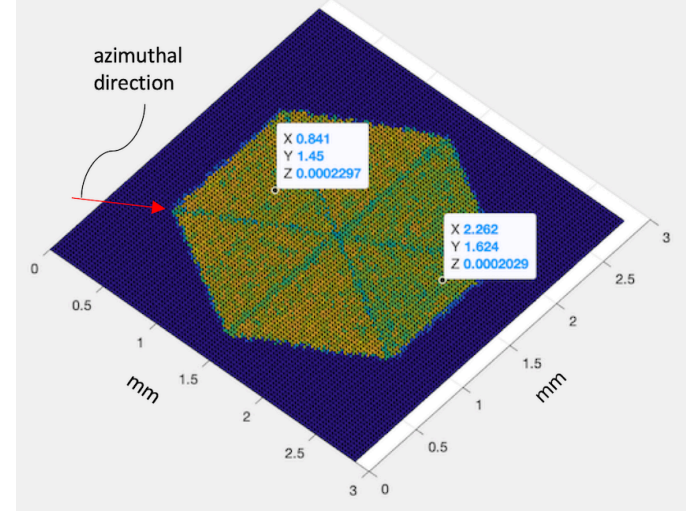

Figure 4a. Flux distribution at focal plane at $5^{\circ}$ incidence.

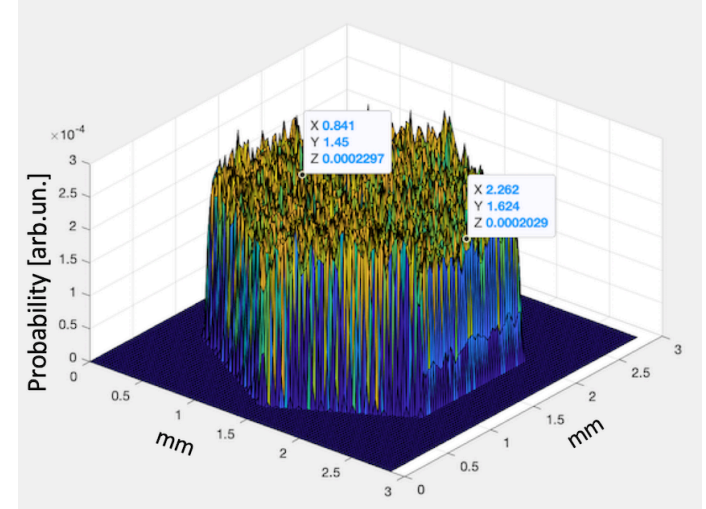

Figure 4b. Flux distribution at focal plane at $5^{\circ}$ incidence.

An even more illustrative is the situation at an incidence angle of $6^{\circ}$ as shown in Figures $5 a, b$.

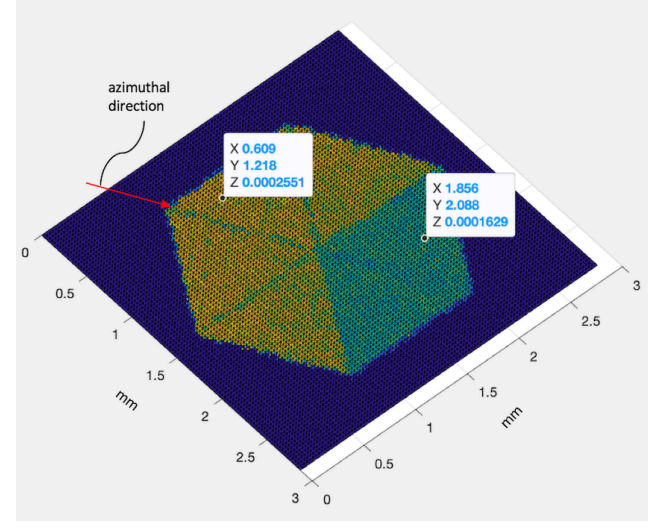

Figure 5a. Flux distribution at focal plane at $6^{0}$ incidence.

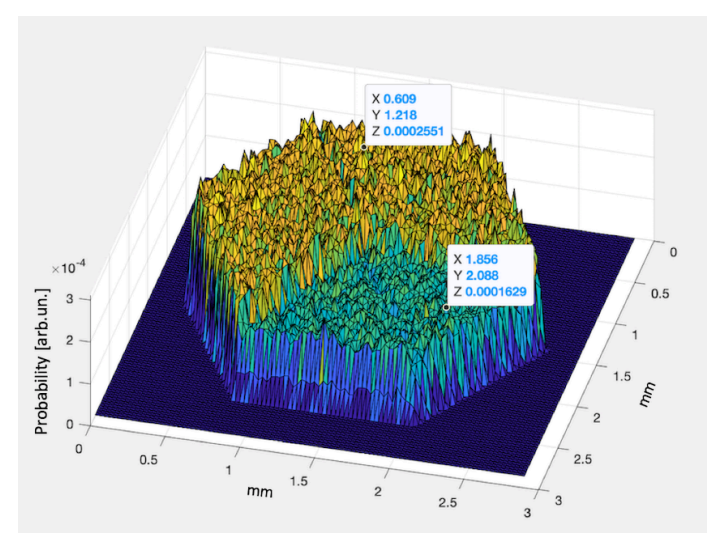

Figure 5b. Flux distribution at focal plane at $6^{\circ}$ incidence.

As seen, the disbalance in the exit flux has now exacerbated. It is also noted that the rejection and the mirror losses at this point are calculated to $1.1 \%$ and $1 \%$ respectively. These losses occur almost exclusively within the OEs farthest along the azimuthal direction. Their magnitudes, however, account only for a minor part of the disbalance which again for the major part is caused by the above mentioned internal redistribution of the flux.

We now take a look at the propagation losses. Assuming absorption of $0.004 \mathrm{~cm}^{-1}$ and an incidence angle of $5^{\circ}$ the absorption losses are calculated to about $6.7 \%$ for the case in Figure $2 \mathrm{~b}$. These losses are spectrally and material dependent but they can be readily estimated since they are additive in nature. For a number of materials this loss is practically zero as is for PMMA in the deep blue spectrum. One final loss which needs to be accounted for is the loss of intensity in the gaps. For the dimensions chosen this loss amounts to $2.8 \%$ but as noted above this loss can be reduced by a proper design of the OEs at the entrance.

Thus, it is concluded that the transmittance of FC concentrators typically varies between $85 \%$ and well above $90 \%$ depending on the materials used and design. This high transmittance is due in part to the high transmission coefficient and in part to the fact that 
they have only one air/solid interface between the solar radiation and the solar cell as the latter is optically connected (through optical matching if needed) to the focal plane. Of course, FC concentrators may be connected serially with other concentrating optics although this generally may result in increased reflection losses at the added air/solid interfaces unless optical matching is provided.

One final inherent advantage of FC concentrators is that off-axis orientation of the solar panel does not produce any damage to any part of the optical system or its neighborhood since in this case direct solar radiation is practically reflected back (rejected) in its entirety. In other words, under no circumstances does any part of the optical system get exposed to a concentrated light flux but the solar cells. For this reason alone, the mirror structure extends all along the whole height of the concentrator to prevent indirect flux from getting absorbed by neighboring surfaces and causing unwanted heating.

\section{Conclusions}

It has been numerically demonstrated that FC concentrators at 100X exhibit sufficiently large acceptance angles and excellent uniformity of the concentrated light flux. Being refractive in nature FCs exhibit absorption losses associated with the materials used. For a typical situation with an absorption of $0.004 \mathrm{~cm}^{-1}$ it is shown that the transmittance of FCs typically exceeds $85 \%$.

\section{References}

[1] I. Katardjiev, A fractal concentrator of direct and indirect light, Clean Energy, 2020, https://academic.oup.com/ce/article/4/4/305/5983284

[2] I. Katardjiev, Optical characterization of a fractal concentrator, https://doi.org/10.33063/diva-430393, 2021

[3] Hébert M., Reflection and transmission of light by a flat interface, Fresnel's formulae. Ph.D. Thesis.Paris, France: Institut d'Optique ParisTech, 2013.

[4] I. Katardjiev, Sun Tracking Method for Fractal Concentrator Panels, https://doi.org/10.33063/diva-433553, 2021 